\title{
ROMPECABEZAS O SIMBOLOS EN EL LENGUAJE NATURAL
}

\author{
Mauricio Molina Delgado
}

\begin{abstract}
This article focuses on the search for an alternative metaphor to explain cognitive phenomena. The frame proposed is that of natural language, together with those prelinguistic structures that are coherent with language itself. Gadamer's hermeneutical circle becomes an evidently linguistic explanatory model. Besides, it seems to encompass neuronal processes. An attempt is made to overcome the contradiction between symbolist and conexionist models by means of a continnum between levels of explanation (semantic and physical). Finally, an attempt is made to readdress Artificial Intelligence on the basis of these considerations.
\end{abstract}

\section{Introducción}

La mayoría de los problemas analizados en las Ciencias Cognoscitivas, tradicionalmente se ciñen a un modelo de arquitectura cognoscitiva que comúnmente no se pone ni siquiera en duda: la idea de que el cerebro es análogo a un computador (Stillings) y que la base material (neuronal o de circuitos) no es relevante (Dennett [81])' Evidentemente todo desarrollo científico está cimentado en premisas no contrastables empíricamente. Sin embargo, en el fondo de esta visión tradicional de las Ciencias Cognoscitivas, subyace una petición de principio. Parece más bien que aquella metáfora (computador-mente) debería ser un posible punto de llegada más que un presupuesto.

La estrategia que sigo en este artículo va precisamente en la dirección inversa. No parto de una metáfora como la del computador, como se hace generalmente. Pienso que resulta imprescindible plantearse nuevos modelos sobre los cuales desarrollar un nuevo paradigma, y que éstos deben partir de lo que efectivamente conocemos empíricamente sobre el fenómeno cognoscitivo y de algunos presupuestos de base que resulten verdaderamente imprescindibles. Es el desarrollo científico mismo el que debe determinar a posteriori la viabilidad de la metáfora computacional.

Hasta el momento conocemos dos características que innegablemente tienen los procesos cognoscitivos que conocemos:

a. Se desarrollan sobre una base biológica (neuronal).

b. El sistema que más claramente expresa los fenómenos congnoscitivos, a pesar de su complejidad, es el Lenguaje Natural (LN) Humano. Otros comportamientos inteligentes, como el de los animales, nos resultan menos transparentes. Las ventajas del LN radican en que a este nivel se pueden expresar las metas y los deseos; se trata de un sistema intencional. Como bien señala Pylyshyn [86], esta característica no puede estar ausente en el estudio de las Ciencias Cognoscitivas.

Un modelo cognoscitivo no debe, entonces, dejar de lado los procesos de cognisción humana, y de hecho debe explicar convincentemente estos procesos mediante el tratamiento correcto del LN. 


\section{Una matáfora alternativa}

\subsection{El concepto simbólico de nivel}

Dennett [81] postula la existencia de tres niveles para comprender el funcionamiento de un sistema cognoscente: un nivel físico, uno de diseño (sintáctico) y otro intencional (semántico). La posición simbolista centra el estudio de las Ciencias Cognoscitivas en un nivel intensional, en cambio, la postura reduccionista-neurológica se coloca a nivel de diseño (Churchland [89]). Otra posición sería la llamada conexionista. Simbolistas como Fodor y Pylyshyn [88] consideran que el conexionismo comete el error de ubicarse a nivel de diseño. Rumelhart y McClelland [86] consideran esencialmente pobre la noción simbolista de nivel. Reconociendo que el nivel al que se trabaja en los modelos conexionistas es ciertamente más bajo $^{2}$, reclaman con razón que éste no es necesariamente un nivel de diseño.

Los niveles de Dennett son de tipo discreto. Mi tesis es que los tres niveles descritos son tendencias en un continuo y que esto provoca que los límites propuestos no siempre sean claros. Son claros en el caso de la computadora, pero difusos en el del cerebro humano.

\subsection{Un modelo hermenéutico}

Gadamer [75] propone un metáfora de los procesos cognoscitivos donde la comprensión se explica por analogía con la lectura de textos, con la ventaja de que ésta última representa en sí misma un proceso de comprensión ${ }^{3}$. Este modelo se denomina Círculo Hermenéutico (Círculo del Verstahen). Gadamer propone una relación dinámica y circular entre el todo y las partes, donde distintos niveles de comprensión se van construyendo, de modo que cada uno de ellos está regido por los supuestos asumidos en el nivel anterior. Cada nivel representa un estado de conocimiento que se ve incrementado por el siguiente, al final del cual es posible que el estado previo sea reelaborado de encontrarse algún problema en su interpretación. Si bien podemos afirmar que cada nivel emerge del marco de posibilidades que le deja el anterior y que se caracteriza por un crecimiento de la comple- jidad, conviene aclarar que esta noción de nivel representa una idea continua donde el emerger es un proceso constante en medio del cual no podemos señalar inicio y final propiamente; es totalmente diferente a los niveles de los que hablan Dennett [81] y Newell [82] donde cada nivel se refiere a la naturaleza de los procesos (físicos, algorítmicos, simbólicos). Caracterizando esta idea continua de nivel, se podría decir que no existe una frontera clara entre diseño, sintaxis y semántica, y que dentro de cada uno de éstos, hay otros niveles de complejidad que se suceden en forma continua.

La doble posibilidad de elaborar desde la idea general a la particular y desde los nuevos estados individuales a la generalidad, es a lo que Gadamer se refiere del siguiente modo:

"La anticipación de sentido que hace referencia al todo sólo llega a una comprensión explícita a través del hecho de que las partes que se determinan desde el todo determinan a su vez a éste" (Gadamer [75], p. 360)

La idea no es novedosa, y se utiliza desde que alguien se interesó por la comprensión de textos. Esto trae como consecuencia la aceptación de visiones de conjunto difusas (regidas por suposiciones) y las posteriores revisiones de dichas visiones, además del carácter lingüístico del proceso cognoscitivo (lectura de un texto o comprensión en general). Pero aun si no se admite este último elemento al pie de la letra, existen múltiples situaciones en que esto se da correctamente. Además, la idea de presupuestos configurando el modelo, revisión del modelo (de los presupuestos) y vuelta a un nuevo modelo con nuevos presupuestos, describe un esquema de círculos que se van construyendo uno sobre otro.

\subsection{El lenguaje como representación}

Winograd y Flores [86] utilizan la idea de Gadamer para juzgar el diseño computacional a la luz de los procesos de comunicación en la práctica social. Voy a profundizar en el significado de la metáfora de los círculos para conocer, junto a Winograd y Flores, diferencias esenciales entre el cerebro y la computadora4. El propósito es profundizar en aspectos concretos que no aparecen en su libro Under standing Computers and Cognition. Sin 
embargo, considero que la postura que defienden es extrema. Ellos despedazan la idea de representación, de la que simbolistas y conexionistas se valen. Mi intención es rescatar una idea más rica de representación que dé cuenta de la naturaleza difusa del lenguaje natural.

Si se quiere, esta representación va a ser pre-lingüística, en el sentido que debe explicar la gramática. Sin embargo, dentro de la idea del continuo se fundamenta un concepto básico del lenguaje natural. La idea de representación que busco sería algo así como un germen lingüístico.

\subsection{Diferencias generales de ambas metáforas}

Si confrontamos ambas metáforas encontramos las siguientes diferencias:

a. Mientras que el modelo simbolista es un modelo lineal y regido por acumulación de datos y ejecución de reglas (en general monotónico excepto por algunos intentos en ese sentido que analizaré en la sección 3.5) el otro muestra una relación circular y fuertemente no monotónica, tanto respecto a su base de conocimiento como a la aplicación de reglas y algoritmos.

b. El primero depende excesivamente de una concepción innatista (ver Chomsky [59]). Bajo el paradigma simbolista de Inteligencia Artificial, un programador llena las estructuras, construye una representación del conocimiento. En las ciencias cognoscitivas la mente requeriría de un "hardware" inusitadamente sofisticado que realice el papel que lleva a cabo el programador en la computadora. Ante la pregunta: "¿Por qué las personas más estúpidas llegan a hablar, mientras que los monos más inteligentes no lo consiguen?", Chomsky responde con dispositivos innatos para la adquisición lingüística (Chomsky y Miller [1963]).

c. Aparte de algunos intentos de Pylyshyn [86] en el paradigma simbolista, la magnificación del papel de lo innato es acompañada de un desprecio por el papel del medio y de la interacción social. d. Presenta un tratamiento rígido de categorización y en general del tratamiento de factores semánticos. Lo mismo que de los procesos de aprendizaje de LN y de las actitudes proposicionales y de los "qualia"6.

e. Para fines de simulación o para fines prácticos, la propuesta que se fundamenta en los Círculos de Gadamer, es inferior.

Esencialmente, la metáfora computacional no da cuenta de los procesos cognoscitivos humanos, más que en aspectos muy reducidos. En la minoría de los casos, el output de un Sistema Computacional concuerda con el del ser humano, pero esto no necesariamente indica que se trata de un buen modelo?

\section{Simbolos y rompecabezas}

A diferencia de Kobsa, quien plantea una clase de equivalencia a la que pertenecen las soluciones humanas y las computacionales, pienso que podríamos describir la relación entre los modelos entendiendo las tareas que pueden ser explicadas mediante la metáfora computacional como uno de los círculos de menor complejidad que se desarrollan en el modelo de Gadamer.

Me voy a valer de una analogía para desarrollar el significado de ambos modelos. Imaginemos a un hombre armando un rompecabezas. Supongamos inicialmente que esta persona actúa de forma lineal colocando una a una las piezas guiado por la forma que éstas tienen, de modo que probablemente en ningún momento tenga que volver atrás (excepto cuando dos piezas sean idénticas en algunos de sus lados. Retomaré este caso al hablar de Razonamiento no Monotónico en la sección 3.5.). Esta imagen corresponde a la Computadora llenando el rompecabezas, guiada por una serie de reglas (forma de las piezas). El caso de un ser humano es totalmente diferente. Inicialmente (asumamos que no se tiene la imagen de la caja del rompecabezas sino que un primer vistazo a las piezas en desorden dan una idea inicial); la persona tiene alguna idea de cómo será la imagen del rompecabezas. Existe una anticipación de la totalidad como la descrita en el círculo herme- 
néutico de Gadamer, de modo que no sólo las reglas "formales" están guiando el proceso de armar el rompecabezas.

Curiosamente el armar un rompecabezas es una tarea no muy diferente de lo que comúnmente hacemos en nuestros actos de conocimiento. Utilizando la imagen del rompecabezas, me voy a centrar en el análisis de cuatro temas centrales en las ciencias cognoscitivas y en las implicaciones que un cambio de modelo debería acarrear.

\section{Procesamiento de Lenguaje Natural \\ 2. Categorización \\ 3. La prueba de Turing \\ 4. Control y representación del conocimiento}

\subsection{Procesamiento del LN}

En general, la visión simbolista entiende el procesamiento del LN mediante el esquema de "grammar driven". Es decir, se postula una preponderancia de la sintaxis en el procesamiento de LN. Una versión poco elaborada consiste en negar totalmente el papel del contexto en el proceso. Sin embargo, es fácil mostrar que muchas oraciones sacadas de contexto pueden ser representadas mediante dos estructuras sintácticas diferentes ${ }^{8}$. Ante este problema se pueden proponer dos estados de procesamiento:

1. Inicialmente se incorpora la oración bajo estructuras puramente sintácticas.

2. En casos de ambigüedad se recurre a principios impuestos por la semántica del LN o por el conocimiento no lingüístico.

El primer estado se describe mediante el "Minimal Attachment Principle" (M.A.P., Clifton y Ferreira) el cual propone un modelo parsimonioso en el que linealmente se adiciona léxico a la estructura, buscando minimizar los nodos del árbol. Esto equivale al rompecabezas armado secuencialmente y además refleja la llamada "Conduit Metaphor" (Reddy [79]), es decir, la suposición dualista de que las formas literalmente son empaques de los significados, y por lo tanto la semántica es independiente de otros niveles lingüísticos, por ejemplo la sinta- xis? . Sólo en casos de ambigüedad se recurre a la regla 2 , que equivale, haciendo la analogía del rompecabezas, a devolverse cuando la forma de un lado de la pieza es igual en dos o más de ellas, de modo que es necesario buscar hacia atrás en un proceso de búsqueda de soluciones. Este "volver atrás" no tiene nada que ver con el Círculo de Gadamer, donde los procesos de revisión se aplican sobre una prefiguración de la totalidad, y no sólo sobre las partes.

La principal debilidad del M.A.P., radica en el hecho de que la secuencia 1-2 es imposible, dado que aun las más simples operaciones del "Parsing" requieren de un sustento semántico. Supongamos la oración "El hombre llamó a su amigo". A pesar de que no existe ambigüedad, el reconocimiento de que "el hombre" constituye un nodo correspondiente a una frase nominal, radica en un proceso paralelo en el que interviene el significado de "el hombre". a la vez que su forma sintáctica. Resulta imposible pensar cualquier estado ( 1 y 2 ) independientemente. En términos del Círculo Hermenéutico podríamos interpretar este proceso como una convivencia del todo y las partes, ya que resulta imposible sintaxis sin semántica. En cierto sentido, podríamos pensar que la semántica en una oración es el todo respecto a la sintáxis. Conviene detenerse un poco en este aspecto: aprehender la semántica de una oración significa comprender lo que la oración denota y connota como totalidad; por otra parte, la sintaxis corresponde a la función gramatical de cada parte de la oración por separado, función que sólo puede entenderse respecto a la semántica de la toda la oración ${ }^{10}$.

Lo que sí existe es una anticipación global de cualquier oración ( y de cualquier texto) en la que se movilizan supuestos y se ponen a prueba. Es útil presentar de nuevo la idea del rompecabezas. Una vez que se empieza a armar, tenemos una idea de la semántica del cuadro (una hipótesis de cómo se vería armado). Las primeras piezas se colocan fácilmente en los bordes, del mismo modo que la función de algunas palabras en la oración se determina rápidamente. De repente aparece una ambigüedad; la evidencia posterior mostrará la colocación correcta. A menudo esto implicará volver atrás, replantearse la imagen global que se tenía. De este modo, se maneja paralela- 
mente el todo (rompecabezas) y la pieza; algo similar sucede con las palabras y su significado y aun con unidades más pequeñas (los morfe- mas, fonemas, etc.) o más grandes (oraciones, párrafos, etc.)

Figura 1

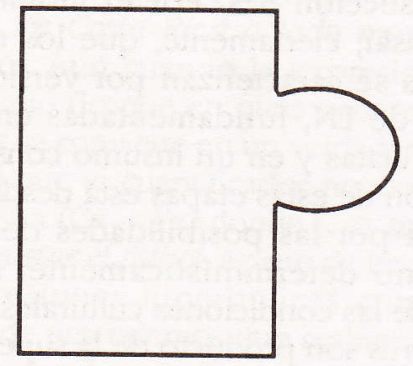

a

Imaginemos el modelo simbolista mediante esta analogía. El proceso de armar el rompecabezas requiere de una serie de reglas que determinen que las piezas a) y b) no se junten (figura 1), mientras que permitan que a) y c) sí lo hagan. Puesto que estas reglas son insuficientes, otras deben referirse al color de las piezas (una especie de semántica de cada pieza) en caso de que más de una pueda calzar en un espacio. La imagen global del rompecabezas (semántica el rompecabezas), no aparece hasta que la última pieza sea colocada. El primer problema que aparece es que la cantidad de reglas para manejar un rompecabezas como un LN sería inmensa, pero sigamos pensando en un rompecabezas relativamente simple.

Un algoritmo como el siguiente sería útil para encontrar una solución:

\section{Rompecabezas (posición)}

Para pieza: $=1 \mathrm{am}$

Si para todo vecino (posición)

Compatible ( pieza, vecino (posición))

luego espacio (posición): = pieza

Rompecabezas (sucesor (posición))

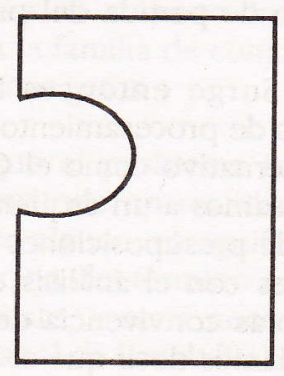

c b

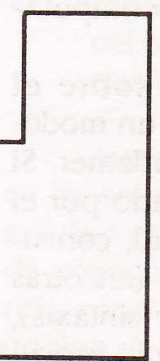

El algoritmo, basado en recursividad y ensayo-error, tiene incluso la propiedad de volver atrás. Bajo este punto de vista parece manejar las exigencias que nos impusimos al pretender partir del Círculo Hermenéutico.

Sin embargo, puesto que el sistema se sustenta totalmente en reglas sobre las piezas (digamos sintaxis), en ningún momento se tienen anticipaciones de la totalidad. Aun cuando el programa ensaya hipótesis sobre la colocación de cada pieza, nunca lo hace sobre el significado global del rompecabezas. Lo que se da es un llenado secuencial hasta que algo falle.

Sin embargo, el modelo tradicional tiene un gran atractivo. Asumamos por un momento sus premisas, donde cada oración es un paquete que contiene significados. El cognoscente recoge la oración y la decodifica colocándola linealmente en una estructura, digamos un árbol, mediante un conjunto de reglas $(r 1, r 2, \ldots r n)$. En caso de que varias reglas se puedan aplicar, se recurre a un conocimiento pragmático que al fin y al cabo podría nuevamente estar formando reglas ( $p 1, p 2, \ldots$ pn), produciéndose finalmente los significados. El modelo es como el siguiente:

Figura 2

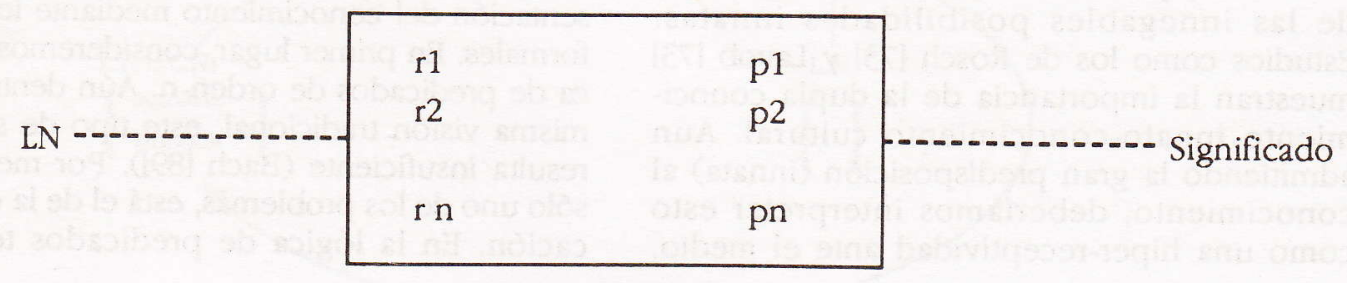

Sistema de Reglas 
Las ventajas del modelo presentado son dos: 1) Tenemos bien identificada la entrada (LN), el procesador (reglas) y la salida (significados). 2) Tenemos además un instrumento para la experimentación, el cual es además el punto de partida del paradigma: la computadora.

Surge entonces la pregunta sobre el modo de procesamiento del LN, bajo un modelo alternativo como el Círculo de Gadamer. Si regresamos a un esquema caracterizado por el uso de presuposiciones de la totalidad, concurrentes con el análisis de las partes (en otras palabras convivencia de semántica y sintaxis), podríamos decir que el LN es su propio procesador. A diferencia de los Lenguajes Formales, el LN es su propio metalenguaje. Este hecho le da su carácter circular a los procesos de cognición. Gadamer habla de que cada palabra es analizada en el contexto de una oración, ésta a su vez en un macrotexto y éste, en medio de una totalidad social e histórica. Además, cada instancia superior se fundamenta en las partes y sus relaciones. ¿Cómo vencer entonces un retroceso hasta el infinito? Está claro que hacia adelante no se divisan más límites y que nuevos horizontes van creciendo sobre suposiciones iniciales y el conocimiento de las partes. Podríamos visualizar círculos cada vez más amplios. El problema es más bien dónde empieza el proceso. Chomsky y los simbolistas han señalado la predominancia de los factores innatos en el hombre y la pobreza del medio en el desarrollo y aprendizaje del LN. Aunque esta posibilidad podría hacernos pensar en el círculo cero $^{11}$ de los procesos cognoscitivos, la verdad es que la premisa de pobreza del medio resulta poco creỉble. Măs bien podemos percatarnos de que el contexto social y lingüístico es muy rico; la ilusión de la pobreza del medio parece provenir de que la mayoría de los procesos de aprendizaje son no supervisados. El caso de los niños salvajes ilustra un medio verdaderamente pobre para el desarrollo del LN y podría analizarse como un fracaso de las innegables posibilidades innatas. Estudios como los de Rosch [73] y Lavob [73] muestran la importancia de la dupla conocimiento innato-conocimiento cultural. Aun admitiendo la gran predisposición (innata) al conocimiento, deberíamos interpretar esto como una hiper-receptividad ante el medio.
Las condiciones innatas permiten a la persona, durante sus primeros años, captar rápidamente muchos aspectos del medio; la gran flexibilidad permite que la información se procese de formas muy diferentes ${ }^{12}$. Retomaré estos problemas en la sección 3.3. Por el momento podríamos pensar, ciertamente, que los estadios tempranos se caracterizan por versiones rudimentarias de $L N$, fundamentadas en las posibilidades innatas y en un insumo constante. La superación de estas etapas está desde un inicio marcada por las posibilidades del LN mismo, pero no determinísticamente, sino dependiendo de las condiciones culturales. Las etapas posteriores son producto de la superposición de nuevos horizonte sobre los más elementales.

\subsection{Semántica y lógica}

Uno de los propósitos de este artículo es mostrar que cualquier lenguaje formal representa solamente un anillo dentro del esquema de Gadamer, y que, por tanto, es insuficiente para caracterizar la semántica de un LN. Tampoco podríamos considerar ese anillo como un Círculo en sí mismo ${ }^{13}$, capaz de dar cuenta de aspectos complejos de la realidad; no podemos pensar en él como la base del modelo. De otro modo, cualquier expresión en LN debería ser representable en LF. En otras palabras, se puede afirmar que éste no se encuentra en las etapas tempranas de desarro1lo, ni como parece desprenderse de la versión simbolista más radicalit, en niveles inconscientes (nivel representacional o "semantical level", según Pylyshyn, "level", o "knowledge level", como lo llama Newell). Si alguna de estas afirmaciones fuera correcta, nuevos anillos podrían desarrollarse a partir de un LF, pero el hecho es que hasta el momento ningún LF parece suficiente para que se desarrolle a partir de él, conocimiento nuevo.

Examinaré algunas posibilidades de representación del conocimiento mediante lenguajes formales. En primer lugar, consideremos la lógica de predicados de orden n. Aún dentro de la misma visión tradicional, este tipo de sistemas resulta insuficiente (Bach [89]). Por mencionar sólo uno de los problemas, está el de la cuantificación. En la lógica de predicados tenemos 
solamente un cuantificador universal $(\forall)$, que se refiere a todos los elementos del dominio, y otro existencial $(\exists)$ para uno o más elementos del dominio. Podríamos agregar un "existe y es único" $(\exists)$, y por extensión tenemos, ningún $x$ $(-\exists \mathrm{x})$ y no todo $\mathrm{x}(\sim \forall \mathrm{x})$. Observemos la expresión En Costa Rica a todo mundo le gusta el fútbol. Aun cuando la expresión identifica el dominio (lo que en otras oraciones proposicionales se convierte en un verdadero problema) es claro que el cuantificador no expresa lo mismo que $\forall \mathrm{x}(\mathrm{Cx} \rightarrow \mathrm{Fx})$ donde $\mathrm{C}$ es ser costamicense y F gustar el fútbol. Aparte de los problemas con expresiones hiperbólicas como la anterior (donde entendemos que realmente no se aplica el predicado a todo el dominio), surge nuevamente la dificultad de expresar cuantificadores difusos como alguin, casi todos, la mayoría, etc.

Tomemos un segundo enfoque desarrollado por Montague [73] y Barwise y Cooper [81]. Consideremos la siguiente oración:

\section{(1) Algunos gatos comen zanaboria}

Sea $\mathrm{A}=$ [come zanahorias]= todas las entidades que comen zanahorias\}.

$\mathrm{B}=[$ algunos gatos $]=\{$ todos los conjuntos que contienen algunos gatos\}

(1) se puede representar como $\mathrm{A} \in \mathrm{B}$. Expliquémoslo del siguiente modo (2):

a. Considérese el conjunto de todas las entidades que comen zanahorias.

b. Considérese el conjunto que contiene como elementos a todos los conjuntos que contienen algunos gatos (en particular) y otros elementos de cualquier tipo.

c. La oración Algunos gatos comen zanahorias es equivalente a decir que el conjunto de los comedores de zanahorias es tal que contiene a todos los gatos que lo hacen y a otros elementos $\left\{g_{1}^{\prime}, g_{2}, \ldots g_{n}, e_{1}\right.$, $\left.e_{2}, \ldots e_{n}\right\}$ donde cada $g_{1}$ es un gato que come zanahorias.

d. De c) se sigue que si los gatos que comen zanahorias pueden clasificarse como algunos gatos, el conjunto descrito debe pertenecer a la familia de conjuntos que contiene algunos gatos.

Aunque parece que el problema inicial se ha solucionado, el método en realidad no ayuda a definir, de modo alguno, el significado de algunos. Una definición del término requiriría considerar el contexto, y sería necesario un tipo de estudio similar al de Lavob [83] en la determinación del significado de cup. El problema es esencialmente un problema de categorización que es tratado por Montague mediante el enfoque tradicional de clases de equivalencia ${ }^{15}$.

Detengámonos en la explicación (2). Se podría decir que el contenido semántico de la formalización $(A \in B)$ está presente en 2). Es más, podemos notar que la formalización hizo uso de expresiones en LN. Aparecen claramente tres aspectos:

1) Los modelos propuestos en LF (sea lógica de predicados o el sistema de Montague) fallan en el procesamiento de al menos una característica del LN (lo hemos visto en el caso de la cuantificación).

2) Sin embargo, el LN es totalmente suficiente para procesar expresiones adscritas a los lenguajes formales analizados (ya lo hemos hecho).

3) Lo más importante es el hecho de que una expresión en LN puede utilizar el mismo LN para ser su propio mecanismo de procesamiento. Obviamente esto no favorece la metáfora computacional. Aquel esquema en el que separábamos

Figura 3

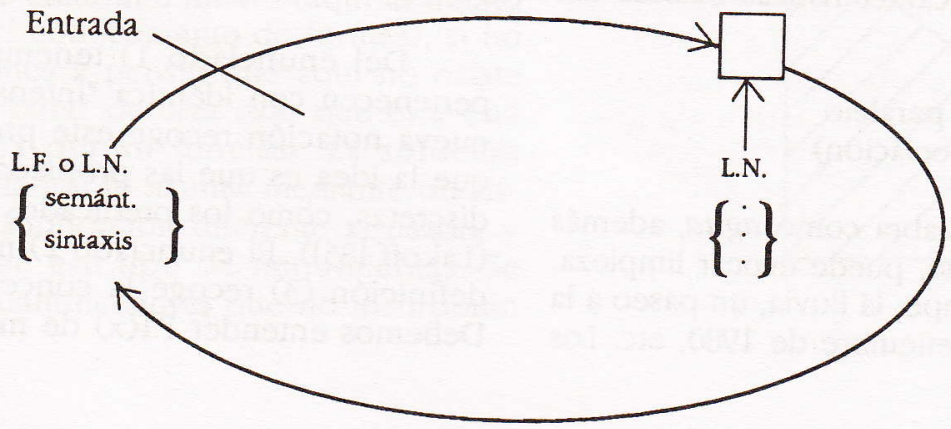


claramente los insumos en $\mathrm{LN}$, las reglas sintácticas y el significado, puede ser sustituido por un esquema circular.

Volviendo al modelo del Círculo Hermenéutico, vemos que éste difícilmente se sostiene si no postulamos que cada anillo y círculo están estructurados lingüísticamente (como lo afirma el mismo Gadamer), en otras palabras, que el propio origen de los círculos es el lenguaje. Así, podemos explicar que el mismo lenguaje formal sea comprendido, ya que éste sí es expresable en LN. De hecho, en la enseñanza de la lógica, nadie llega a comprender $\mathrm{P} \rightarrow \mathrm{Q}$ mediante la tabla de verdad, sino mediante el conocimiento de que "cuando el evento P se cumple, Q necesariamente es verdad, aunque de ser $P$ falso no se sabe nada Q" o "es inconcebible que $Q$ sea falso si $P$ es verdadero", o bien mediante algún ejemplo expresado en LN. El LF es un círculo de la cadena, algo así como un subconjunto.

Sin embargo, el problema de la cuantificación no es único. Otra dificultad que comúnmente es dejada de lado es la referente a los "qualia". El problema está íntimamente ligado al del sentido. Sin embargo, la única aproximación posible dentro de un lenguaje formal es la que le da Frege por medio del concepto de mundos posibles, lo cual es una simplificación extrema. Existen oraciones que mantienen un sentido y en las que un mundo que las soporte no podría seguir este sistema. En un poema de Robert Lowell aparece la siguiente frase: "Pregunto dónde está Carolina y Carolina eres tú", que podría considerarse como la convivencia de P y no P (Carolina no está aquí y por eso pregunto donde está, pero Carolina está aquí). Sería ridículo negar que existe algún sentido en la frase.

Una última consideración respecto de la comparación entre el LN y cualquier LF, es que éste carece de dos características básicas del cerebro humano:

\section{a. Procesamiento paralelo \\ b. Integración (asociación)}

Una simple palabra como agua, además de tener un referente, puede evocar limpieza, frescura, sed, el tiempo, la lluvia, un paseo a la playa, el día 3 de setiembre de 1980, etc. Los niveles semánticos parecen ser infinitos y además altamente integrados. El esquema típico de sentido-referente-significado debe resignarse a la verdad.

\subsection{Categorización}

La noción clásica de categorización, descansa sobre el mismo esquema de acumulación lineal guiada por reglas que he representado mediante la imagen del hombre o la máquina que arma un rompecabezas sin una referencia dinámica a la totalidad. De acuerdo con esta postura definamos la categoría C como la clase de equivalencia $\mathrm{C}=\{\mathrm{C} 1$, $\mathrm{C} 2, \ldots, \mathrm{C} n\}$, construida mediante una relación $\mathrm{R}$, tal que $\mathrm{C} 1, \mathrm{Cj}$ que pertenezcan a $\mathrm{C}$. La definición anterior se fundamenta únicamente en las unidades elementales de la clase de equivalencia $\mathrm{C}$ y en las propiedades que permiten agrupar a esas unidades C1 en una sola clase.

Esencialmente me interesan dos aspectos que se deducen del modelo precedente:

1) Sean $\mathrm{C} 1, \mathrm{Cj}$ cualesquiera elementos de $\mathrm{C}$, $\mathrm{C} 1$ es tan elemento de $\mathrm{C}$ como $\mathrm{Cj}$. Por tanto, toda propiedad de los elementos de $C$, se predica con igual intensidad sobre $\mathrm{C} 1$ y sobre $\mathrm{Cj}$.

2) Las reglas que definen $C$ son libres de contexto y son independientes de la subjetividad del individuo que categoriza (i.e son objetivas).

Conviene introducir la notación de la Lógica de Predicados de Primer Orden para explicar el punto. Podemos redefinir $\mathrm{C}$ del siguiente modo:

$$
\begin{aligned}
& C=\{x / P 1(x), P 2(x), \ldots, P n(x)\} \\
& \text { a R b } \leftrightarrow[P 1(a) \wedge P 2(a) \wedge \ldots \wedge P n(a)] \wedge \\
& {[P 1(b) \wedge P 2(b) \wedge \ldots \wedge P n(b)]}
\end{aligned}
$$

Del enunciado 1) tenemos que a y b pertenecen con idéntica "intensidad" a C. La nueva notación recoge este presupuesto, ya que la idea es que las propiedades de $\mathrm{C}$ son discretas, como los predicados $\mathrm{P} 1(\mathrm{x}), \ldots \mathrm{Pn}(\mathrm{x})$ (Lakoff [851). El enunciado 2) muestra que la definición (3) recoge la concepción clásica: Debemos entender P1(x) de modo que si ' $y$ ' 
es una variable sobre el dominio de todas las personas, ' $y$ ' no ocurre libre en P1, presumiblemente acotada por el cuantificador universal. Luego, todas las personas perciben P1, del mismo modo.

El problema de esta concepción deriva del hecho de que las propiedades reales son continuas y no discretas. El enfoque simbolista supone no obstante que aun siendo continuas en el mundo real, la función de la categorización consiste en convertirlas en discretas para ser manejables.

Un segundo problema viene del cuantificador universal que se introduce para considerar a los sujetos que categorizan. Me parece que a esta posición subyace la idea de un fuerte factor innato en la adquisición de LN, específicamente en cuanto a la semántica, lo cual parece, en principio, verse fortalecido por el estudio de Rosch [73] en el que se demuestra (al menos en cuanto a categorización de colores y formas) un elemento innato. La conclusión de dicho estudio es que existen algunos prototipos naturales ${ }^{16}$ y que aun cuando algunas culturas difieren en su modo de categorización, siempre existe la misma predisposición fisiológica a un solo sistema de categorización de colores y formas. Berlin y Kay [69] muestran algunos patrones jerárquicos, de modo que ciertos colores de mayor rango reciben denominación en el lenguaje siempre que todos los de menor jerarquía la tengan. El punto aquí es que, a pesar del factor fisiológico, grupos humanos como los dani utilizan un sistema de categorización diferente "no natural", lo que nos hace presumir un segundo elemento importante, de orden cultural. A un resultado similar llega Lavob [73] al encontrar los dos factores importantes en la determinación del significado de la categoría "taza" (observe que ésta no es una clase natural): su función y su forma. Esto podría asimilarse en los mismos factores cultural e innato (aquí lo innato podría ser el conocimiento de formas), si no nos detenemos a pensar que aquí no existe una "forma pura" de taza sino que ésta está determinada por su función. La solución parece ser definir el asunto mediante un sistema de cuantificación diferente al usado, y el LN provee ese tipo de herramientas. Se requieren cuantificadores que no incorporen todos los elementos del dominio y que identifiquen cierta fuerza de pertenencia. Sin embargo, ya vimos los serios problemas que éstos presentan para el esquema simbolista. Lo mismo podría decirse del problema de los "qualia" y de la inclusión del factor subjetivo en la categorización.

Un nuevo problema que Lavob [73 ] señala al enfoque tradicional es el del supuesto de independencia:

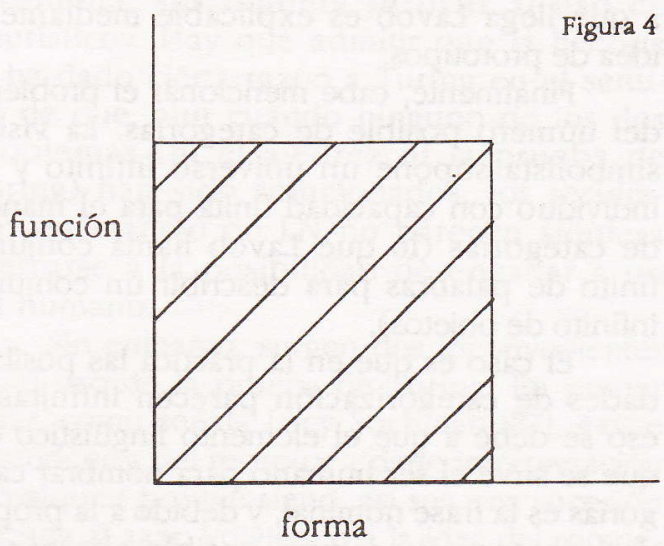

El modelo de la figura 4 (clásico) podría describirse así:

$P(o b j e t o=t a z a)=$

o si $x>q$ o $y>s$

1 en otro caso

La figura 5, muestra en cambio, probabilidades que dependen de ' $x$ ' $y^{\prime} y^{\prime}$

$$
\begin{aligned}
& P(\text { objeto }=\text { taza })=f(x, y) \text { donde } \\
& f(x, y)=g(a x+b y+e)
\end{aligned}
$$

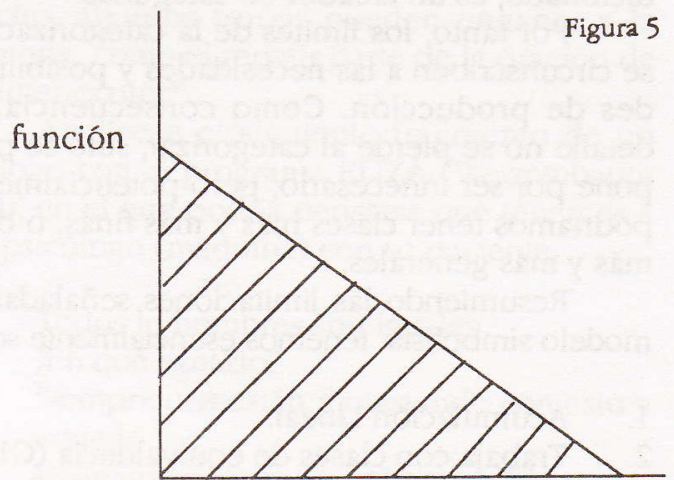

forma 
En el experimento de Lavob, parece que el modelo de la figura 5 resulta el más indicado. Sin embargo, estrictamente hablando, el modelo sigue suponiendo independencia. Sería importante realizar estudios en regresión para determinar en cuáles otros casos se aplica un modelo donde

$f(x, y)=g(a x+b y+c x y+e)$, donde el término cxy denota interacción. En realidad el modelo a que llega Lavob es explicable mediante la idea de prototipos.

Finalmente, cabe mencionar el problema del número posible de categorías. La visión simbolista supone un universo infinito y un individuo con capacidad finita para el manejo de categorías (lo que Lavob llama conjunto finito de palabras para describir un conjunto infinito de objetos).

El caso es que en la práctica las posibilidades de categorización parecen infinitas, y eso se debe a que el elemento lingüístico del que se sirve el ser humano para nombrar categorías es la frase nominal, y debido a la propiedad de recursividad es posible abarcar un dominio infinito" ${ }^{17}$. Así, las siguientes son frases nominales que denotan categorías:

\section{Los leones}

Los leones amarillos

Los leones del zoológico

El león que vi el 25 de mayo de 1990 en el zoológico.

Existe en el ser humano una capacidad constructiva respecto de la categorización; aun aceptando que ya viene fisiológicamente condicionado, es un creador de categorías.

Por tanto, los límites de la categorización se circunscriben a las necesidades y posibilidades de producción. Como consecuencia, el detalle no se pierde al categorizar, sólo se pospone por ser innecesario, pero potencialmente podríamos tener clases más y más finas, o bien más y más generales.

Resumiendo las limitaciones señaladas al modelo simbolista, tenemos esencialmente seis:

\section{Acumulación Lineal.}

2. Trabaja con clases de equivalencia $\left(C 1, C_{j}\right.$ pertenecen con igual intensidad a $\mathrm{C}$ ).

3. Independencia respecto del contexto.
4. Propiedades discretas.

5. Negación de la incertidumbre que introduce el elemento subjetivo.

6. Definicción fundamentada en la forma pero no en la función.

Yo pretendo que toda esta problemática es perfectamente tratable mediante el enfoque que he venido defendiendo.

Procediendo del mismo modo que lo he estado haciendo, busquemos ejemplos de la cognición humana. Wittgenstein toma la categoría "juego", encontrando que ninguna propiedad se cumple a lo largo de los elementos que aparentemente le pertenecen. A lo más que podemos aspirar es a un esquema reticular que enlaza un elemento con otro y a éste a su vez con un tercero, etc., sin que ningún predicado se aplique a todos los elementos. Este esquema se conoce como "parecido familiar" y utilizando las matrices de Lavob, puede ser representado así:

elemento

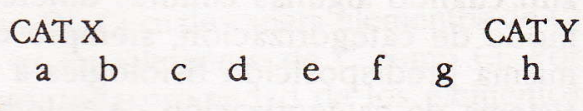

pro-

pie-

dad

$$
\begin{array}{lllllllll}
1+ & + & + & + & + & - & + & - \\
2 & - & - & + & - & - & - & - & - \\
3 & + & + & + & + & + & - & + & - \\
4 & + & + & - & + & - & + & - & - \\
5+ & + & + & + & + & - & - & - \\
6 & - & - & - & - & - & - & - \\
7 & + & - & + & + & + & - & - & -
\end{array}
$$

En este caso no podemos marcar un límite preciso entre las categorías X y Y, ni tampoco pretender una jerarquía al modo de Rosch. En el caso de una categoría como "juego", se pueden dar inserciones que muestren aún más diluidos los posibles límites, por ejemplo los juegos de video, como apunta Lakoff.

Tanto las categorías por prototipos como las de parecido familiar, comparten una importante propiedad: se parecen a aquel rompecabezas a medio armar en el que no se utiliza un sistema basado en reglas. Al contrario, en ambos casos se tiene una idea difusa de la totalidad (la categoría) y no un análisis formal (basado en reglas) de las piezas (los elemen- 
tos) ${ }^{18}$. En el caso de los prototipos, tenemos "mejores ejemplos", algo así como piezas claves que dan una imagen más clara del paísaje que estamos armando. En cuanto al parecido familiar, el "paisaje" es como una gradación de colores: una pieza se asemeja a su vecina, y ésta a su vez a la siguiente, aún cuando este parecido no sea transitivo, como un pastizal donde dos piezas alejadas sean una muy clara y otra muy oscura, pero donde la transición sea suave entre piezas contiguas.

No se trata de defender ninguna modalidad de categorización como una teoría general: hace falta un enfoque más ecléctico. Así como en dyirbal existe una categoría cultural que incluye mujeres, fuego, cosas peligrosas, pájaros y otros animales, otra como "número natural" en occidente parece cumplir, efectivamente con el modelo tradicional (obsérvese que eso no elimina el factor cultural: en algunas culturas, el cero resulta desconocido y en otras se categoriza como número). El punto aquí es el que se ha estado defendiendo desde el principio según el modelo del círculo hermenéutico: el ser humano domina los procesos formales sólo como uno más de los anillos de la comprensión (ni siquiera parece ser fundamental que lo haga), el cual está sobre otros principios generales de tipo lingüístico. Abusando de nuevo del término, la categorización clásica es sólo un subconjunto ${ }^{19}$ de nuestro sistema de categorización real.

\subsection{La Prueba de Turing}

En 1950, Allan Turing propuso el juego hombre-mujer como prueba para juzgar si una máquina era inteligente. La naturaleza del juego consiste en que una persona en una habitación debe descubrir por medio de preguntas cuál es el sexo de dos personas que están en otra habitación (un hombre y una mujer). Turing asegura que en condicione normales los interrogados pueden ser suficientemente ágiles, de modo que quien interroga no alcance seguridad de haber adivinado. La idea de Turing es sustituir a uno de los dos que están en el cuarto por una máquina. Si la máquina desarrolla correctamente cierta astucia y capacidad de "mentir", estaría exhibiendo comportamiento inteligente.
A primera impresión, la prueba tiene la virtud de no proponer labores complejas, pero que en realidad no muestren, necesariamente, un comportamiento inteligente. Por ejemplo, el caso del ajedrez, donde la labor implica manejo inteligente en el ser humano, pero donde si tuviéramos una máquina con suficientes recursos, el problema se podría solucionar por fuerza bruta mediante un árbol de búsqueda, sin siquiera recurrir al uso de heurísticos. Hay que admitir que la historia le ha dado cierta razón a Turing en el sentido de que, aun cuando ninguno de los dos problemas (ni el ajedrez ni la prueba de Turing) han sido solucionados, los resultados en manejo de LN no parecen siquiera acercarse a la posibilidad de engañar a un ser humano.

Sin embargo, surgen dos inconvenientes en el uso de la prueba de Turing. En primer lugar, como señala Lucy Suchman [87], existe la tendencia del receptor a darle interpretación a cualquier posible signo, así sea una sucesión lanzada al azar. Volviendo a la idea del rompecabezas, uno esperaría que las piezas representaran una imagen, pero supongamos que en el conjunto existen piezas sin ninguna relación (de varios rompecabezas, por ejemplo). La persona que lo arma podría pasarse un rato pensando en alguna idea inicial de totalidad hasta que alguna evidencia lo lleve a concluir que no existe tal. Del mismo modo, la persona que realiza la prueba de Turing podría interpretar, aun cuando el manejo del LN no fuera correcto, que del otro lado existe una persona algo excéntrica, alguien que no sabe escribir en la computadora o algún extranjero. De hecho, algunos trucos pueden engañar a la persona, como el repetir parte de la oración de su interlocutor ${ }^{20}$.

Analícese el siguiente fragmento de un diálogo con el programa ELIZA (Weizembaum [66]), en el que podría pensarse que tenemos a un psicólogo (máquina) con su paciente:

P: Todos lo hombres son iguales

M: ¿En qué sentido?

P: $\quad$ Siempre nos están molestando con esto y aquello

M: Podría darme un ejemplo específico

P: Bueno, mi novio me hizo venir aquí

M: Su novio la hizo venir aquí 
P: $\quad$ El dice que estoy deprimida a cada rato

M: Siento mucho que esté deprimida

P: $\quad$ Es verdad, estoy triste.

Existen al menos tres inconsistencias en las intervenciones de la máquina que una persona podría interpretar como correctas. En primer lugar, una frase como "todos los hombres son iguales" es un tópico, de modo que la pregunta siguiente "¿En qué sentido?" es extraña. Se podría esperar tal vez algo como "¿Por qué piensa eso?", y de hecho a esto es a lo que el paciente responde. El recurso de repetir lo que el paciente dijo ("Su novio la hizo venir") parece correcto en el contexto, pero generalmente iría acompañado de un cambio en la entonación, de modo que se agregue un contenido semántico (admiración, decirle al paciente que tenga calma, etc.). Al darse la conversación mediante la pantalla, la frase resulta al menos sospechosa. Finalmente, al decir el paciente "El dice que estoy deprimida a cada rato", la contestación "Siento mucho que esté deprimida" muestra incomprensión del tiempo verbal (no es ahora), y de que sólo se trata de la opinión del novio. Entonces, la suposición del paciente es que, de hecho, el doctor descubrió que era cierto ("Es verdad, estoy triste"). A pesar de las inconsistencias, podría pensarse que a estas alturas el paciente no se da cuenta de la situación hasta que se dé un error verdaderamente grave. Aun cuando en la actualidad ningún programa se acerca a lograrlo, si algún día alguno pasara la prueba debe tomarse esto en cuenta.

El segundo problema de la prueba de Turing es el que señala Searle [80]. Supongamos que en efecto diseñamos un sistema que maneje el LN y pase la prueba, por ejemplo, uno capaz de corregir los problemas señalados en el fragmento de la interpretación con ELIZA. Aun así, no necesariamente estaría comprendiendo la situación. Sería similar a una persona que recibe, en un cuarto cerrado, preguntas en chino y produce respuestas en chino mediante una serie de reglas en español que enseñan a manipular los símbolos en chino. Searle argumenta que no se podría afirmar que el individuo comprende chino ${ }^{21}$. Afirmarlo y aceptar la prueba de Turing, equivale a pensar que la comprensión es mera manipulación de símbolos como puede desprenderse de la visión tradicional.

Si regresamos a reorganizar el panorama mediante el Círculo de Gadamer, debería proponerse una nueva prueba que determinara la inteligencia de cualquier entidad.

Recurriendo a la imagen del Círculo, podríamos ver la inteligencia como la capacidad que tiene una entidad para pasar de un estado de conocimiento y un cuerpo de suposiciones a otro diferente, quizá más amplio y hasta contradictorio con el anterior. Además debemos agregar que cada estado involucra una visión de totalidad que sería, en el caso del argumento del cuarto chino, todo aquello que no se explica mediante la simple manipulación formal. Mi propuesta sería que aceptemos que una entidad es inteligente si es capaz de reformular su cuerpo de conocimiento. De ahí se desprende que si una máquina tiene la posibilidad de desenvolver en un LN y es verdaderamente inteligente, debe ser capaz de aprender otro LN totalmente diferente (cualquier LN). Una máquina a la que se le haya dado la posibilidad de manejarse en español, mediante la formalización del conocimiento gramatical y con el uso de ciertos heurísticos que la ayuden a simular semántica, debería ser capaz de aprender una segunda lengua. Estrictamente hablando, la prueba de Turing es correcta en el sentido de que tarde o temprano un sistema como el descrito, sin un verdadero manejo semántico, debería fallar y sería incapaz de acondicionarse a un contexto cambiante en el que los círculos hermenéuticos crecen de modo continuo. Sin embargo, si algunas reglas probabilísticas tuvieran suerte en un momento dado, y tomando en cuenta la tendencia de buscar un sentido a todo en el individuo receptor, es posible que un sistema así pasara la prueba.

Otra ventaja de la prueba propuesta es que elimina la confusión común entre inteligencia de la máquina e inteligencia del usuario o inteligencia del programador. En el caso del cuarto chino, dos personas efectivamente saben chino: el que provee las reglas (programador o la fuente de donde obtiene esas reglas) y el usuario. La confusión máquinausuario ya ha sido comentada, pero en el caso máquina-programador no existe confusión ya que el problema es demasiado complejo para 
que el mismo programador lo domine. Tanto la máquina inteligente como el programador son capaces de aprender cualquier $\mathrm{LN}$, pero ninguno de los dos es capaz de dar cuenta de una formulación que incorpore un LN cualquiera ("vivo" o "muerto"). Lo que está detrás de todo es la anticipación de la totalidad a la que se refiere Gadamer (la semántica) y el argumento es que no se puede adquirir una lengua sin poseer una semántica prefigurada en otro LN, o en algún rudimento de LN. En cierto sentido, el todo se muestra anterior a las partes. No me refiero por semántica sólo a la significación de palabras individuales o su relación con el mundo (referencia). En el aprendizaje de idiomas extranjeros se aprehende algún sentido de las frases antes que el significado individual de las palabras, y ambos significados se van afinando mutuamente.

Una importante ventaja que tiene esta propuesta sobre la de Turing, es que ésta última no posee medios para dar cuenta de la inteligencia animal. Un experimento como el conducido por Allen y Beatriz Gardner [65-72] con la chimpancé Washoe, en el que se le enseñó el American Sign Language (Ameslan), muestra un nivel de inteligencia muy superior al de cualquier programa de computación ${ }^{22}$. Sin embargo, estos animales estarían aún más lejos de pasar la prueba de Turing que la misma conversación con ELIZA. El experimento de los Gardner puede ser considerado como una versión simplificada de la prueba que propongo. Si aceptamos la evidencia, el uso por parte de algunos chimpancés de términos como "pájaro de agua" para referirse a un pato, "manzana de color anaranjado" para denotar una naranja o " comida que duele y hace llorar" para hablar de un rábano, parece existir un crecimiento del horizonte semántico, similar, aunque ciertamente mucho más reducido del que implica el aprendizaje de una segunda lengua.

\subsection{Control y representación}

Ya hemos discutido los problemas con los que se enfrenta la representación de conocimiento mediante formalismos lógicos, sea la lógica de predicados o sistemas más poderosos como el de Montague. Otras estructuras de representación de conocimientos son las redes semánticas ${ }^{23}$. En general se podría describir una red semántica como un conjunto de nodos que representan cada uno un concepto, con conexiones que representan relaciones entre nodos. El principal problema de esta representación es que no provee un marco para el fenómeno del aprendizaje, y más bien los conceptos se consideran entidades hechas (símbolos). En otras palabras, no se puede pensar en éste como un modelo de la mente. No provee de un método para ampliar los círculos de significado de la cognición. El tipo de relaciones que describe una red puede ser del tipo de IS-A o ISPART (es un ..., es parte de ...) de modo que cualquier intento de proveer un esquema de categorización fallaría en simular un esquema de prototipos. En el esquema de clases de equivalencias, un elemento es parte (ISPAR'T) o no es parte de una categoría; en el esquema de prototipos, donde las relaciones no son de tipo discreto, los elementos son parte en diferentes grados. Las dos relaciones que se representan en la figura 6 mediante una red semántica, son exactamente iguales.

Figura 6

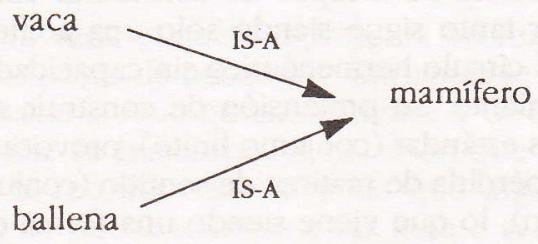

Una posibilidad de trabajar con prototipos es darle una ponderación a las relaciones de pertenencia, pero esto seguiría sin dejarnos una clara explicación del fenómeno, ya que no se está dando una extracción natural del prototipo, sino que se están copiando los patrones del comportamiento humano. El problema sigue siendo un problema de aprendizaje.

Las redes semánticas, utilizadas para el procesamiento de LN, son básicamente iguales a los árboles o "phrase makers" utilizadas para dar cuenta, dentro de algunas teorías lingüísticas, de la sintaxis de una oración. Las relaciones definidas son del tipo sujeto, agente, beneficiario, etc., así que el problema de llenado ${ }^{24}$ e interpretación de oraciones en LN, se encuentra con los mismos problemas en el 
tratamiento de ambigüedad que cualquier estructura que requiera de ser llenada mediante la aplicación secuencial de reglas.

Los marcos ("frames") (Minsky [75]) son otro tipo de representación que pretende aprovecharse de relaciones de jararquía para un manejo más dinámico de información incompleta, y así sustentar cierto tipo de aprendizaje. Sin embargo, su forma es esencialmente la misma que una red semántica en cuanto a su inflexibilidad para auto-corregirse (al hablar de métodos de control retomaré con detalle el punto).

Schank [73 y 75] propone una representación llamada "conceptual dependency" (CP) para el manejo generalizado de LN. El propósito del CP es inferir sobre oraciones una representación independiente del lenguaje en que fue originalmente expresada, utilizando un vasto número de relaciones más elaboradas que IS-A o ISPART: por ejemplo, tiempo verbal, transferencia de posesión, movimiento, etc.. CP es un intento mayor de representación semántica; sin embargo, sigue apegado al patrón tradicional en el que el todo se construye sólo a partir de sus partes, en el sentido de que se trabaja con unidades pequeñas de significación. Es incapaz de considerar contexto y por tanto sigue siendo sólo una anillo dentro del círculo hermenéutico sin capacidad de crecimiento. Su pretensión de construir significados estándar (conjunto finito), provoca además la pérdida de matices de sentido (conjunto infinito), lo que viene siendo una forma de desechar los "qualia" a nivel lingüístico.

Los "Scripts" (Schank [77]) representan un tipo de estructura que conviene analizar más a fondo. Se trata de secuencias estereotipadas de eventos en un determinado contexto como puede ser la visita a un restaurante. El "script" debe declarar las condiciones, propiedades, roles, metas, etc., de los eventos, de modo que el patrón de comportamiento quede determinado.

El esquema de "script" corresponde a la filosofía racionalista occidental y puede ser descrito como un plan, una secuencia de actos que van de un estado inicial a uno meta guiados por intenciones y reglas.

"A plan is any hierarchical process in the organism that can control the order in which a sequence of operations in to be performed. [...]
A plan is for the organism, essentially the same as a program for a computer" (Miller, Galanter, Pribram, [60]).

Queda establecida de nuevo la metáfora de la computadora. Una de las primeras aplicaciones artificiales de este modelo de planificación, fue puesta en práctica a principios de los años 60 en la construcción de un robot llamado Shakey, que caminaba por varios cuartos evitando obstáculos y trasladando algunos objetos. El programa de Shakey se denominó "Strips". A la hora de trasladar un modelo como éste a una explicación de la conducta, surge el problema de explicar la interacción social: los seguidores de esta idea de planificación extienden el modelo a dos o más entidades que crean planes propios y reconocen los del otro.

La idea de una secuencia guiada por metas parece tener dificultades para explicar intenciones que nunca se realizan o imprevistos, como el caso del que frena por un niño que se atraviesa. Remediar el problema mediante reglas que manejen excepciones plantearía una explosión del tamaño de la estructura. Surgen también problemas para explicar intenciones en las que no se traza un plan y en el estudio de acciones inconscientes. Suchman [87] plantea la posibilidad de que más que un mecanismo generativo, los planes sean un artefacto de nuestra razón para encasillar acciones. El hecho de que B esquematice las acciones de $\mathrm{A}$ en un plan no implica necesariamente que A haya manejado de ese modo la información. Se puede tener en cuenta que una acción puede ser ejecutada de muchas formas y que el curso de los eventos que generan la acción puede ser analizado "a posteriori", aunque no predicho. Schmidt, Sidharan y Goodson [78] identifican como patrones del proceso de conocimiento de planes, la construcción de una hipótesis simple sobre el rango de posibles planes, la selección de los posibles eventos para poner a prueba la hipótesis, y finalmente la revisión de la hipótesis de acuerdo con los eventos. La semántica y la pragmática parecen ser los ámbitos en los que se pueden inferir planes posibles. Este esquema ha resultado de alguna utilidad para la representación de conocimientos específicos (como en el caso de Shakey), pero los problemas del sentido común parecen resistirse al análisis. En este caso, el dominio bajo el que se da cuenta de 
las acciones parece infinito y adscrito a "scripts" cada vez más profundos que expliquen el paso de una situación a otra debido a distracciones, obstáculos o errores.

La idea de planes recomienda un tratamiento secuencial sobre los procesos de control. El giro que he estado proponiendo implica el regreso a estructuras cambiantes. Suchman propone el concepto de "acciones de situación" para caracterizar los cambios y revisiones sobre la marcha, siendo de nuevo ésta una versión del círculo hermenéutico. El principio es que todo curso de acción depende de las circunstancias materiales y sociales. Este modelo no sólo describe fenómenos como el del automovilista que va hacia adelante sin una definición clara de su itinerario, sino también el proceso mediante el cual un interlocutor ordena la evidencia e infiere un plan que describa la situación de acción (de nuevo se trata de un anillo en una cadena creciente). Las acciones en situación carecen de reglas estáticas, y las representaciones por medio de planes ocurren cuando actividades antes transparentes, se vuelven problemáticas. Como resulta evidente, este tipo de procesos se ejecuta dentro del LN.

Los planes son sólo trazos de orientación para situaciones en evolución, como el plan de juego que sigue un equipo de fútbol. Este tipo de esquema no permite predecir el curso de la situación. De nuevo prepondera la visión del rompecabezas sobre las piezas individuales, pero esta situación está permanentemente en cambio.

Podríamos decir que el mecanismo de control mediante los "scripts", correspondiente a la idea de "plan", es monotónico. Los planes son descripciones secuenciales de posibles acciones que nunca varían. La naturaleza de nuestros procesos cognoscitivos requiere de un tratamiento no monotónico, que nos obliga a buscar un mecanismo diferente, o al menos más amplio, que el de los planes de acción. Conviene revisar los sistemas que han sido diseñados para manejar razonamientos no monotónicos. Podemos inicialmente estudiar un mecanismo de control como el que mencionamos cuando se habló de un algoritmo para llenar rompecabezas. Conocemos este método como "backtracking" y consiste en un procedimiento recursivo que permite recorrer el árbol de búsqueda, de modo que si se encuentra una solución el proceso pare, y en caso de no obtener solución, busque cambiar la última rama hasta agotar las posibilidades (profundidad primero). Supongamos que el problema de buscar una fecha y hora para una reunión se soluciona de este modo (Rich 83 ).

\section{El proceso es el siguiente:}

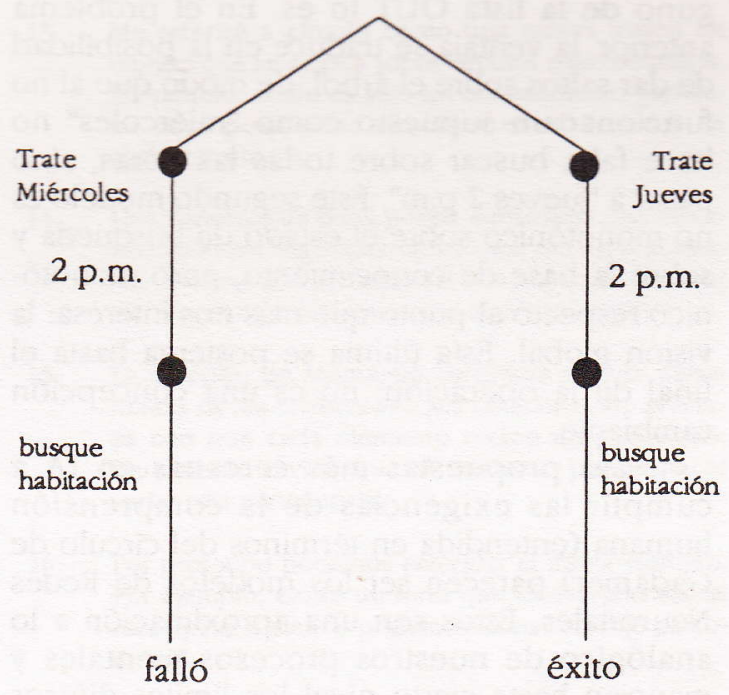

Aquí, cada etiqueta representa en realidad varios pasos de búsqueda. Inicialmente se trata de hacer la reunión un miércoles, y de ser esto posible se buscarán las horas en que es factible la reunión. En caso de que algún miembro no pueda reunirse a esa hora o si no hay lugar de reunión, se tratará de buscar en otros días (jueves), y se repetirá el proceso. Existe aquí un nivel de razonamiento no monotónico, puesto que el sistema busca improvisando posibilidades hasta que se encuentra con una contradicción. Digamos que el proceso es no monotónico sobre la búsqueda en la estructura, pero no hay posibilidad de hacer variaciones sobre la base de conocimiento. El otro problema que se había señalado es que el proceso está basado en un cuerpo rígido de reglas como en el caso del individuo que pretende armar el rompecabezas sin imaginarse el paisaje. El sistema se puede describir como no monotónico sobre el estado de búsqueda, pero monotónico sobre la base de conocimiento y en cuanto a la visión de totalidad. 
Stalman [77], propone un sistema dinámico sobre la base de conocimiento, basado en la idea de asumir aquello que puede ser verdadero como verdadero hasta que no se demuestre lo contrario, en cuyo caso se modifican los supuestos en la misma base de conocimiento. Cada suposición será verdadera si todos los elementos de su lista IN son verdaderos y ninguno de la lista OUT lo es. En el problema anterior, la ventaja se traduce en la posibilidad de dar saltos sobre el árbol, de modo que al no funcionar un supuesto como "miércoles" no hace falta buscar sobre todas las horas, sino pasar a "jueves 2 p.m". Este segundo modelo es no monotónico sobre el estado de búsqueda y sobre la base de conocimiento, pero monotónico respecto al punto que más nos interesa: la visión global. Esta última se posterga hasta el final de la operación; no es una concepción cambiante.

Las propuestas más cercanas en IA a cumplir las exigencias de la comprensión humana (entendida en términos del círculo de Gadamer) parecen ser los modelos de Redes Neuronales. Estos son una aproximación a lo analógico de nuestros procesos mentales y manejan hasta cierto nivel los límites difusos de lo cognoscible. A diferencia de las ideas simbolistas, aparece en el conexionismo una posible explicación de la génesis de los conceptos. Del mismo modo que la idea del círculo hermenéutico, trata con la creación y ampliación de los conceptos ${ }^{25}$. Además, el conexionismo puede utilizar la idea del todo y las partes en forma análoga a como la hemos estado presentando ${ }^{26}$. Por ejemplo, en el reconocimiento de patrones difusos en palabras escritas es posible reconocer la palabra con partes borrosas mediante el análisis paralelo de las palabras existentes en la base de conocimiento, de las letras, y de las subpartes de cada letra (rasgos) (Rumelhart y Mac Clelland [861). De este modo, es posible una labor que fallaría de llevarse a cabo mediante el análisis de las partes (digamos las letras) una a una. Sólo una suposición de la palabra hace posible reconocer la letra borrosa. La ventaja de las redes neuronales sobre otros esquemas de representación es que hacen posible que los concepto emerjan, a diferencia de las redes semánticas que asumen dicho nivel como si estuviera dado. Sin embargo, el precio que se paga es la enorme maraña de relaciones que dificultan el trabajo a nivel de oraciones o unidades más grandes. Desde un punto de vista pragmático, el conexionismo pierde herramientas poderosas con las que cuenta el simbolismo, por ejemplo la recursividad. Minsky [91] señala la necesidad de un enfoque ecléctico que considere, según la situación, el nivel simbólico o el neuronal, como correctos para el análisis. Mi punto de vista es que podemos ver el nivel simbólico como un círculo superior en el esquema de Gadamer, y que la metodología de escalar y descender en los niveles de complejidad es el mejor modo de entender el fenómeno. Sin embargo, las formas de representación del conocimiento con las que contamos no han alcanzado niveles de manejo que expliquen la génesis de la "totalidad" que es necesario suponer para comprender el fenómeno. En el futuro, nueva información sobre el funcionamiento del cerebro podrá llevarnos a mejores formas de representación.

\section{Conclusión}

Partir de las cosas mismas, el objeto de estudio mismo debe ser el imperativo de cualquier ciencia ${ }^{27}$. De este modo, las Ciencias Cognoscitivas deben partir de su propio objeto y deshechar presupuestos de base que puedan dificultar su desarrollo. El resultado de esta operación es cambiar una serie de conceptos sobre el significado de inteligencia, aprendizaje, etc., y sobre las metodologías que deben seguirse. Esto no significa acabar con la computadora como posibilidad de desarrollo, pero sí asumir qué tipo de dirección debe seguir el desarrollo de sistemas. Las implicaciones de este paso serían dejar mucha de la comodidad de modelos parsimoniosos, de la claridad de conceptos, desafiar la "navaja de Ocam". El círculo hermenéutico de Gadamer parece indicar que las líneas de investigación deben centrarse en el procesamiento en paralelo y el razonamiento no monotónico. Las limitaciones que encuentran los modelos de redes neuronales y los sistemas de razonamiento no monotónico, no opacan las grandes potencialidades que tienen. Sin embargo, debemos comprender la necesidad de un enfoque holístico. No se trata de una especie de misticismo, como algunos 
piensan; se trata de una especie de "teoria del campo unificado", que comprende los niveles de análisis no como países con fronteras definidas, sino como parajes con diferentes alturas y diferentes climas. Lo que quiero decir es que los símbolos son de una complejidad, pero no de una naturaleza diferente a las conexiones neuronales, y que de hecho, existe intercambio de los niveles físicos y los conceptuales ${ }^{2 \mathrm{i}}$.

Mi propuesta no es reduccionista, en el sentido de que reconozco niveles de complejidad que no podemos explicar desde un nivel físico, pero mi propósito ha sido romper el "fetichismo de los símbolos" que ha dominado la teoría cognoscitiva, y que se cae por su propio peso con el análisis del lenguaje.

\section{Notas}

1. Me referiré a ésta como la posición "simbolista". Dennet, Fodor y otros utilizan el nombre "funcionalista", pero éste se presta a confusión debido a que existe una postura lingüística que usa este mismo nombre.

2. En el mismo sentido que el Lenguaje de máquina es de menor nivel que el de Pascal o el Prolog.

3. De este modo se evita la posibilidad de caer en interpretaciones absurdas como las que ocurren al extrapolar algunos elementos de la computadora a la interpretación de la mente.

4. A diferencia de ellos, me dedicaré a aspectos específicos de esta diferencia. En el estudio mencionado, los autores se centran en la generalidad de la interacción social.

5. Los símbolos denotan objetos y relaciones entre ellos. El problema es expresar deseos o creencias. Aun cuando éstos pueden estar en forma proposicional, se pierde la referencia en un mundo objetivo.

6. Los "qualia" son los sentimientos asociados a estos estados mentales. Parece imposible derivar "qualias" de un sistema físico de símbolos.

7. Por ejemplo, el caso de las operaciones aritméticas es claramente insuficiente para suponer que el modelo es bueno.

8. Por ejemplo, Stillings presenta la siguiente: "The clerk called all the customers on the phone list".

9. Por cuanto la distinción tajante de los pasos 1 y 2 supone esta separación.

10. Winograd y Flores probablemente dirian que el análisis sintáctico se da sólo en condiciones de breakdown.
11. Me refiero a que bajo la idea de Gadamer en ningin momento se parte de nada. Siempre existe algün "background" por simple que sea. El círculo cero sería algo así como un background implantado en el disenio.

12. Por ejemplo, las diferencias en los sistemas de categorización de los colores. Incluso la predisposición innata no siempre corresponde al sistema empleado, como se deduce del estudio de Rosh [73]

13. Me referiré a círculo como una nueva visión de mundo que se amplia sobre círculos más reducidos. En cambio, anillo es sólo un conocimiento particular cuya posibilidad descansa en el dominio de algún círculo.

14. Una versión más débil podría considerar una representación no lingüistica como base del nivel simbólico. Minsky [91] presenta así una visión de consenso respecto a la polémica con el conexionismo.

15. La solución de Montague considera sólo la pertenencia de los elementos a sus conjuntos, no la fuerza con que cada elemento recibe un predicado. Igualmente olvida que el contexto puede hacer variar esta pertenencia.

16. Por prototipo podemos entender el mejor ejemplo. Por ejemplo, existe un tono que es considerado el más típico; a éste lo podemos llamar el "rojo prototípico".

17. Esto porque dentro de una frase nominal puede existir otra frase nominal, y a su vez dentro de ésta puede haber otra, y así hasta el infinito.

18. Las categorias son difusas aun cuando los prototipos sean definidos, puesto que no hay ninguna regla para determinar en qué punto termina una categoría y empieza otra.

19. En el sentido de que del total de formas en que categorizamos, una parte, efectivamente, responde a este modelo.

20. En pacientes con afasia severa, esta estrategia de repetir partes de la oración hace que parezca que el paciente sólo está un poco distraído pero entendiendo la conversación.

21. Algunos como Hofstadter [82] no aceptan el experimento mental de Searle. Esencialmente, Hofstadter critica la noción de "entender" que utiliza Searle y considera imposible realizar exitosamente dicho experimento.

22. No todos aceptan la evidencia de este experimento. La réplica es que probablemente los experimentadores cometieron el mismo error de dar significado a lo que no lo tiene.

23. Esta y oras formas de representación son modificaciones o ampliaciones de la lógica de predicados. 
Comparten el espíritu simbolista en el hecho de que sus elementos son símbolos de los objetos y relaciones.

24. Es decir, la introducción de una parte de la oración de un nodo específico del árbol.

25. Lo que he llamado el círculo cero de la cognición podría ser equiparado a una red neuronal con un alambrado básico pero desprovista de conceptos aprendidos. Siempre tenemos un "background", aun al principio.

26. Esto no quiere decir que todas las versiones del conexionismo satisfacen al modelo de Gadamer.

27. La historia muestra como las ciencias han frecuentemente perdido ese norte. Así, la economía y la sociología nacieron más apegadas al modelo biológico que a su mismo objeto. Sin embargo, la búsqueda del objeto debe seguir siendo un imperativo.

28. Churchland [89] describe como el ejercicio continuo que lleva al aprendizaje, provoca cambios físicos en la constitución sináptica de las neuronas involucradas.

\section{Bibliografia}

Bach, E. 1989. Informal Lectures on formal semantics. Nueva York. State University of New York Press.

Barwise, J. y R. Cooper. 1981. "Generalized quantifiers and natural language". Linguistics and Philosophy 4, 159-219.

Berlin, B. y P. Kay. 1969. Basic color terms: Their universality and evolution. Berkeley y Los Angeles: University of California Press.

Clifton, C. y F. Ferreira. (por aparecer). Modularity in sentence comprebension. In standing. Cambridge, Mass.: MIT Press.

Chomsky, N. 1959. "Nerbal Behavior", Language, vl. 35, pp. 26-58.

Chomsky, N. y G. A. Miller. 1963. "Introduction to the Formal Analysis of Natural Languages". Handboook of Mathematical Psychology, vol. II pp. 269-321.

Churchland, S.1989. Neurophilosopby. Massachusetts. MIT Press.
Dennett, D. 1981. Brainstorms.

Fodor, J.A. y Z.W. Pylyshyn. 1988. "Connectionism and Cognitive Architecture" En Pinker, Steven y Jacques Mehler (88) Connections and Symbols. pp. 4-48.

Hofstadter, D. y D. Dennett, eds. 1982 The Mind's I: Fantasies and Reflections on Self and Soul. Bantam Books.

Lakoff, G. 1985. Women, fire and dangerous things.

Lavob, W. 1973. The boundaries of words and their meanings.

Minsky, M. 1975. "A framework for representing knowledge". En P.H. Hinton, ed., The psychology of computer vision. New York, McGraw-Hill.

Minsky, M. 1991. "Logical Versus Analogical or Symbolic Versus Connectionist". A I Magazine, pp. 34-51.

Montague, R. 1977. "The proper treatment of quantificationin ordinary English". En Thomason, R., ed., Formal philosophy. New Haven, Conn.: Yale University.

Newell A. 1982. "The Knowledge Level". Artificial Intelligente 18: pp. 87-127.

Pylyshyn, Z. 1986. Computation and Cognition. Massachusetts MIT Press.

Reddy, M. 1979. "The conduit metaphor - a case of frame conflict in our language about language". En A. Ortony, ed., Metaphor and Thought. Cambridge: Cambridge University Press.

Rich, E. 1983. Artificial Intelligence. Singapur.

Rosch, E. 1983. On perceptual structure of perceptual and semantic categories.

Rumelhart, D. y J. McClelland. 1986. Parallel Distributed Processing. 
Schank, R. 1973. "Identification of Conceptualizations Underlying Natural Language". En Computer Models of Thought and Language, R.C. Schank \& K.M. Colby (Eds.), Freeman, San Francisco.

Schank, R. 1975. Conceptual Information Processing. North-Holland, Amsterdam.

Schank, R. y Abelson. Scripts, Plans, Goals, and Understanding. Erlbaum, Hillsdale, N.J.

Searle, J. "Minds, Brains and Programs". The Bebavioral and Brain Science 3: pp. 416-456.

Stallman, R. y G. Sussman. 1977. "Forward Reasoning and Dependency-Directed.
Backtracking in a System for Computeraided Circuit Analysis". Artificial Intelligence, vol. 9, No. 2.

Stillings, N., et. al. Cognitive Science: $A n$ Introduction.

Suchman, L. 1987. Plans and situated actions. Cambridge University Press.

Turing, A. 1950. "Computing Machinery and Intelligence". Mind; reimpreso en 1964, Minds and Machines, ed. A.R. Anderson. Englewood Cliffs, N.J.: Prentice-Hall.

Wittgenstein, L. 1953. Philosophical Investigations. New York: Maxmillan. 